\title{
Relações entre produção cientifica e políticas públicas: o caso da área da saúde dos povos indígenas no campo da saúde coletiva
}

\author{
Relationships between scientific production and public policies: the \\ case of indigenous people's health in the field of collective health
}

Juliana Fernandes Kabad (https://orcid.org/0000-0002-9471-6418) ${ }^{1}$

Ana Lúcia de Moura Pontes (https://orcid.org/0000-0001-9162-5345) ${ }^{1}$

Simone Monteiro (https://orcid.org/0000-0003-2009-1790) ${ }^{2}$

${ }^{1}$ Escola Nacional de Saúde Pública Sérgio Arouca, Fiocruz. Leopoldo Bulhões 1480, Manguinhos. 21041 210 Rio de Janeiro RJ Brasil.julianakabad@gmail. com

${ }^{2}$ Instituto Oswaldo Cruz, Fiocruz. Rio de Janeiro RJ Brasil.

\begin{abstract}
This paper analyses the relationship between studies on the health of indigenous people in public health and public policies aimed at reducing ethnic-racial inequalities. This selection assumes that scientific production on the subject is part of the societal effort to confront health inequities and guarantee the rights and public policies of indigenous people. In total, 3,417 papers were found between 1956 and 2018, and 418 were selected for analysis from systematic literature mapping in the PubMed/Medline, Scopus, Lilacs, Sociological Abstract, and Web of Science databases. Initially, the literature is marked by the biomedical benchmark. After 1990, publications and dialogue with the human and social sciences are expanded, including the analysis of the implementation of indigenous health policy. We identified that the knowledge produced is associated with the political, social, and scientific transformations of the health reform and the indigenous agenda. Scientific production increased in 2010. We can conclude that the knowledge guiding the scientific production on indigenous health was established from a horizon politically implicated with the studied populations and improved Indigenous Health Subsystem.
\end{abstract}

Key words Indigenous health, Collective health, Health inequities, Systematic mapping, Brazil
Resumo Este artigo analisa as relações entre os estudos sobre a saúde dos povos indígenas na saúde coletiva e as políticas públicas voltadas para redução das desigualdades étnico-raciais. Tal recorte parte do pressuposto de que a produção científica sobre o tema integra o esforço societário de enfrentamento das iniquidades em saúde e garantia dos direitos e políticas públicas em saúde dos povos indígenas. A partir de mapeamento sistemático da literatura nas bases Pubmed/Medline, SCOPUS, Lilacs, Sociological Abstract e Web of Science, foram localizados 3.417 artigos entre 1956 a 2018 e selecionados 418 para análise. Inicialmente a literatura é marcada pelo referencial biomédico, mas após 1990 amplia-se o número de publicações e o diálogo com as ciências humanas e sociais, incluindo a análise da implementação da política de saúde indígena. Identifica-se que o conhecimento produzido está associado às transformações politicas, sociais e científicas da reforma sanitária e da pauta indigenista. A partir de 2010 há um aumento da produção científica. Conclui-se que o conhecimento que baliza a produção científica sobre saúde indígena foi se constituindo a partir de um horizonte politicamente implicado com as populações estudadas e o aprimoramento do Subsistema de Saúde Indígena.

Palavras-chave Saúde indígena, Saúde coletiva, Iniquidades em saúde, Mapeamento sistemático, Brasil 


\section{Introdução}

A temática da saúde dos povos indígenas passou a compor a agenda de preocupações de diferentes instituições e atores sociais no Brasil, de modo mais sistemático e contínuo, há quatro décadas. Na pauta do Estado, quando passou a se responsabilizar pelas políticas públicas de atenção à saúde aos povos indígenas. No campo acadêmico pela produção de conhecimento científico em diversas áreas do conhecimento. $\mathrm{Na}$ arena jurídica e de luta por direitos, protagonizada pelos movimentos indígenas e pelo terceiro setor, com a reivindicação de direitos à assistência integral e pública à saúde, entre outros aspectos. Nesse cenário pós-Constituição de 1988, a pauta da saúde indígena é considerada fundamental para a consolidação da cidadania e dos direitos destes povos originários na sociedade brasileira.

Existem 305 etnias indígenas no território brasileiro, com uma população aproximada de 900 mil pessoas segundo o último Censo Populacional realizado em $2010^{1}$. Mesmo que quantitativamente consista em $0,43 \%$ do conjunto da população brasileira, representa significativa diversidade sociocultural ${ }^{2}$. Por consequência, inúmeros são os desafios para o enfrentamento das desigualdades e vulnerabilidades dessas populações, como a consolidação da cidadania, equidade e direitos constitucionalmente garantidos ${ }^{3-6}$.

Para compreender as iniciativas voltadas para a saúde dos povos indígenas, é preciso referir que a Constituição de 1988 ampliou a responsabilidade do Estado com a saúde pública da população em geral com a criação do Sistema Único de Saúde (SUS) ${ }^{7}$. O SUS tem como propósito a universalização da assistência à saúde de forma equânime, pública e gratuita nos níveis primário, secundário e terciário a todos os cidadãos do território brasileiro e em consonância com as demais áreas de atuação do Estado ${ }^{8,9}$. Como resultado de articulações de movimentos indígenas e indigenistas, movimento sanitarista, pesquisadores e agentes públicos, ao longo das décadas de 1980 e $1990^{10}$, foi criado em 1999 o Subsistema de Atenção à Saúde dos Povos Indígenas, incorporado ao SUS, por meio da Lei no 9.836 .

O Subsistema tem por objetivo primordial oferecer atenção primária em saúde nos territórios indígenas, com base nas suas especificidades socioculturais, linguísticas e geográficas, bem como propiciar aos indígenas o acesso aos níveis secundário e terciário da rede pública de saúde ${ }^{11}$. A rede de atenção à saúde é regulamentada pela Política Nacional de Atenção à Saúde dos Povos
Indígenas (Portaria no 254/2012) e se organiza por meio de 34 Distritos Sanitários Especiais Indígenas, tendo como diretriz a atenção diferenciada.

Ainda que se reconheça os avanços neste modelo para a garantia da universalidade do acesso dos povos indígenas aos serviços oficiais de saú$\mathrm{de}$, estudos apontam as recorrentes dificuldades na implementação da atenção diferenciada e efetiva participação indígena na elaboração, execução e avaliação do subsistema ${ }^{12,13}$. Nessa direção, cabe destacar que, concomitante à organização do Subsistema, a saúde dos povos indígenas foi institucionalizada no Brasil enquanto temática de produção científica, sendo crescente a sua visibilidade nacional e internacional ${ }^{14}$.

Ressalta-se que a organização do campo do conhecimento da saúde coletiva no Brasil, devido às suas características epistemológicas, pragmáticas e políticas ${ }^{15}$, alinhado às reinvindicações do movimento sanitarista e consolidação do SUS, possibilitou um espaço propício para o desenvolvimento científico sobre desigualdades étnico -raciais em saúde ${ }^{16,17}$ e, em especial, sobre a saúde dos povos indígenas ${ }^{18,19}$. Segundo Paim ${ }^{20}$, a saúde coletiva é um campo do saber constituído para iluminar a complexidade do processo saúde-doença, considerando suas diferentes dimensões e implicações na realidade social, a partir da crítica a uma perspectiva reducionista de saúde.

Compreende-se que a produção científica compõe um dos elementos necessários para a caracterização de um campo científico e que essa dinâmica envolve a participação de diferentes atores sociais e inúmeras composições epistemológicas, políticas e pragmáticas. Bourdieu ${ }^{21}$ define a ciência como um campo de construções, alinhamentos e embates nos aspectos simbólicos, epistemológicos e ideológicos, mediados pelo capital científico, articulados pelos atores que compõe e atuam nessa estrutura ${ }^{22}$. Tal estrutura é marcada por dinâmicas heterogêneas e assimétricas, entre áreas centrais e periféricas, em termos de poder de influência e impacto no próprio campo científico e demais campos (político, econômico, cultural, entre outros ${ }^{23}$. Assim, a articulação dos pesquisadores e suas redes de pesquisas possibilita o desenvolvimento e o aumento da abrangência e circulação do conhecimento científico, não somente na arena científica, mas para outros setores da sociedade ${ }^{24}$.

Seguindo esse eixo de análise, este artigo orientou-se pela prerrogativa de que analisar o percurso da produção sobre a saúde indígena possibilita revisitar paradigmas, conceitos e ações 
que sustentam não somente a atividade científica, mas também as políticas públicas. Diante dos atuais ataques e retrocessos nos direitos indígenas ${ }^{25}$, o mapeamento sistemático da literatura nacional e internacional sobre o tema nos oferece elementos para refletir sobre a produção do conhecimento científico e a sua relação com o contexto social e político mais amplo. Desse modo, visa iluminar as iniquidades e desigualdades étnico-raciais em saúde e ampliar o escopo de atuação e interlocução das ciências em relação a realidades sociais vulneráveis.

\section{Metodologia}

No período de maio a agosto de 2018 foi realizada um mapeamento sistemático da literatura científica sobre a saúde dos povos indígenas no Brasil em periódicos nacionais e internacionais. A escolha exclusiva por artigos visa compreender a circulação de ideias difundidas e utilizadas como instrumento primordial para comunicar evidências científicas em âmbito global ${ }^{26}$, ainda que não seja o único elemento que define a produção científica. Por meio do levantamento das publicações objetiva-se caracterizar a dinâmica do comportamento da produção em determinado campo do saber ${ }^{27}$.

A busca foi realizada em cinco bases de dados consideradas estratégicas no armazenamento e difusão de artigos no campo das ciências da saúde, humanas e sociais, quais sejam: "PubMed/ Medline”, "SCOPUS”, "Lilacs", "Sociological Abstract" e "Web of Science", com busca ampla e sem definição de período. As estratégias de busca foram orientadas pelos Descritores em Ciências da Saúde (DeCS) adaptados aos critérios de cada base, em torno dos termos Health indigenous peoples, Indigenous population e Brazil. Optou-se pela terminologia em inglês, dado que é o idioma padrão de busca das bases. Com exceção da base Pubmed/Medline, utilizou-se uma combinação de descritores pré-definidos da própria base, denominada Medical Subject Headings (MeSH) que abarca: Indians, south american (MESH) AND Brazil (all terms).

A partir das estratégias de busca foram localizados 3.417 artigos científicos. Os critérios de seleção foram aplicados a partir da leitura do título, do resumo e de palavras-chave, avaliando se os artigos: (1) abordavam populações indígenas localizadas no Brasil e (2) se trabalham com temáticas associadas à análise das condições de vida e saúde das populações indígenas e políticas pú- blicas de saúde indígena. Dessa forma, foram excluídos 1.210 artigos duplicados; 853 artigos que não se referiam ao contexto brasileiro; e 936 artigos que não se referiam a temática de saúde dos povos indígenas. Assim, foram selecionados 418 artigos para análise das seguintes variáveis: ano, periódicos, autor, título, palavras-chave e resumo.

\section{Resultados}

Os 418 artigos foram publicados em 173 periódicos brasileiros e estrangeiros, no período entre 1956 e 2018. Apenas 13 publicações não indicavam o periódico. A maior concentração de artigos vincula-se aos Cadernos de Saúde Pública, com 22\%, seguido pelo periódico da Organização Pan-Americana de Saúde (OPAS) com 4\%. Os demais artigos estão distribuídos em diferentes periódicos, com variação entre $3 \%$ a $1 \%$ do total, sendo 35\% distribuídos em 148 periódicos, muitas vezes com apenas um artigo em casa revista, conforme Gráfico 1.

Ao observar a distribuição temporal do quantitativo de publicações, verifica-se um crescimento após a década de 1990, com um salto exponencial nos anos 2000 a 2009 e entre 2010 e 2018 (N = 211). Esse último período desponta com o maior quantitativo de artigos científicos, conforme Gráfico 2.

No Gráfico 2 chama a atenção que até a década de 1970 há poucos registros de artigos científicos. Mas, isso não significa a ausência de produção científica em períodos anteriores, como sugere o estudo de Buchillet ${ }^{28}$. Esta obra, consiste na maior revisão bibliográfica sobre o tema, tendo reunido 3.222 títulos de estudos sobre povos indígenas no Brasil e de países vizinhos, desde resumos em eventos científicos, artigos científicos, cartilhas, teses, dissertações, livros e capítulo de livros, em diferentes eixos temáticos.

No presente levantamento, o artigo mais antigo foi publicado em 1956 na revista Sociologia. Intitulado Convívio e contaminação. Efeitos dissociativos da população provocada por epidemias em grupos indígenas, trata da problemática das contaminações de doenças que ameaçavam a sobrevivência de populações, sendo um dos primeiros estudos na área de demografia indígena. Seu autor, Darcy Ribeiro, foi um dos grandes antropólogos e representante público no Congresso Nacional a reivindicar pelos direitos dos povos indígenas no país ${ }^{29}$.

Nas décadas 1950 a 1960, período em que as ideias "evolucionistas" ainda influenciavam 


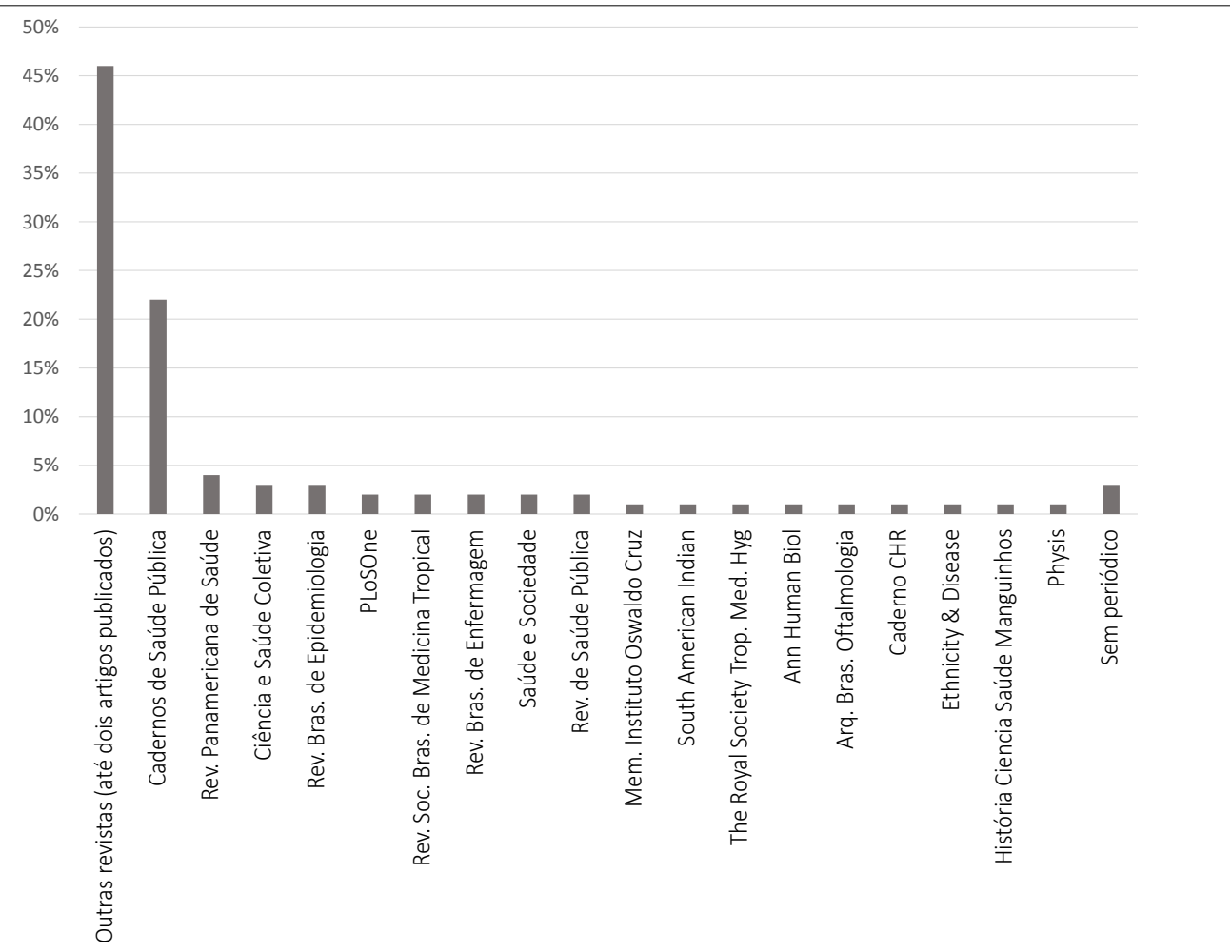

Gráfico 1. Distribuição $(\mathrm{N}=418)$ da produção científica de 1956 a 2018 por periódico científico.

Fonte: elaboração própria.

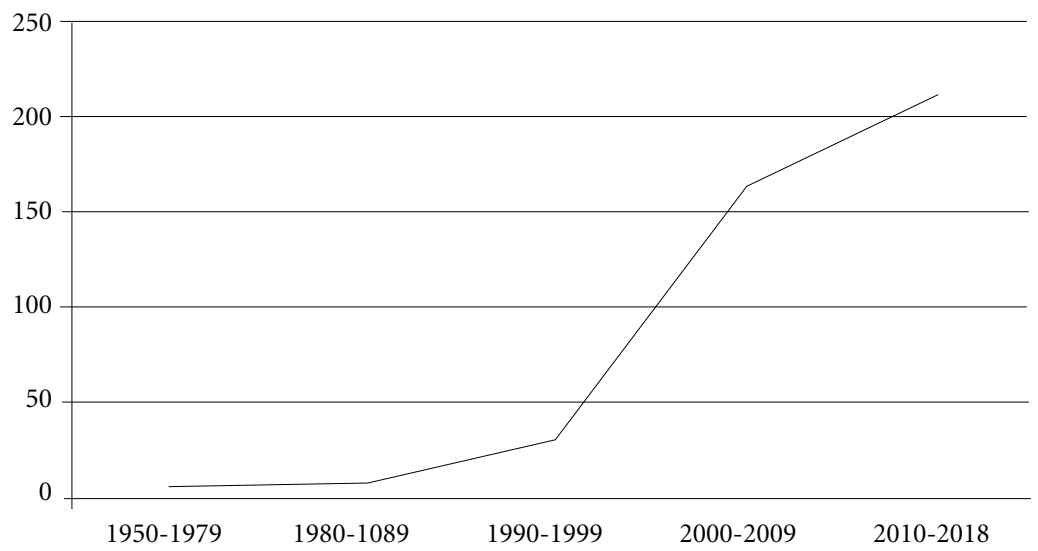

Gráfico 2. Distribuição temporal das publicações por décadas, desde 1950.

Fonte: elaboração própria.

as pesquisas biológicas, havia um interesse em pesquisas junto aos povos indígenas em todo mundo ${ }^{28}$ centrado na compreensão e no enfrentamento das grandes epidemias que assolavam essas populações. Todavia, há poucos registros de artigos científicos, por ser uma modalidade que se tornou mais propagado por meio de periódicos científicos mais recentemente. Ainda nesse 
período nota-se o foco na verificação do grau de especificidade ou generalidade dos povos indígenas com relação a outras populações. Segundo Souza et al..$^{30}$, estudiosos, precursores em genética de populações indígenas, estavam preocupados em construir um conhecimento capaz de explicar as características genéticas das populações humanas. A escolha por povos indígenas ocorria por serem identificados como povos "puros" do ponto de vista biológico. Assim, os geneticistas não tinham uma preocupação prioritária com a doença, que surgia como um fator seletivo para a condição de sobrevivência genética ${ }^{30}$.

A partir da década de 1970 são mais presentes os estudos na área de medicina tropical sobre os agentes etiológicos que provocavam agravos mórbidos em regiões de clima tropical; área que permanece em constante desenvolvimento. Importante mencionar que a emergência dessa temática ocorre em meio às ações desenvolvimentistas na região amazônica no período da ditadura militar brasileira, que geraram diversos impactos negativos aos povos indígenas como mortes, epidemias e espoliação de seus territórios ${ }^{31}$.

Na década de 1980, com a emergência do campo da saúde coletiva, diversos temas do conhecimento passam a figurar, como o acesso das populações indígenas aos serviços públicos de saúde, marcado pela realização da $1^{\text {a Conferência }}$ Nacional de Proteção à Saúde do Índio, em 1986. A partir da década de 1990 aumenta em cerca de $20 \%$ as publicações em periódicos científicos em comparação com a década anterior. Esse crescimento coincide com um período intenso de debates sobre a inclusão dos indígenas no SUS e o início do surgimento dos primeiros grupos de pesquisa no país que estudam a saúde dos povos indígenas. Entre 1990 e 1999 as publicações abordam assuntos variados, focados principalmente nas condições epidemiológicas das populações indígenas.

Na década de 2000-2009, período da implantação do Subsistema de Saúde Indígena no SUS, há um elevado aumento do número de publicações, que corresponde a $38 \%$ do total da revisão. Estes achados corroboram os resultados de Teixeira e Silva ${ }^{31}$ acerca da produção de teses e dissertações centradas na saúde indígena. As autoras identificaram um salto quatro vezes maior da produção acadêmica em relação à década de 1990 a 1999, ainda que o enfoque do trabalho fosse no campo da antropologia no Brasil. As temáticas prevalentes nesta década são, com base na primeira palavra-chave do artigo, nutrição, mortalidade infantil, tuberculose, política na- cional de saúde, sistemas médicos tradicionais, saúde mental, participação social, saúde bucal, interculturalidade, entre outros.

Sobre os periódicos, a tendência da década de 2000 segue a mesma da revisão como um todo, referido no Gráfico 1, com a maior concentração (30\%) na Revista Cadernos de Saúde Pública. O salto quantitativo nos anos 2000 pode ser compreendido pela edição especial do periódico Cadernos de Saúde Pública sobre a temática, em 2001, constituída por 16 artigos; $41 \%$ do que se produziu pela mesma revista na década e $13 \%$ do total das revistas nesse período. Para uma análise mais contextual, cabe referir o editorial desse número, que ocorre no âmbito dos debates dos 500 anos da colonização portuguesa, como ilustrador das preocupações científicas e pragmáticas a respeito do tema da saúde indígena em meio a estruturação no Subsistema dentro de SUS a partir de 1999:

Mesmo que transbordem evidências quanto às condições de marginalização sócio-econômica, com amplos impactos sobre o perfil saúde/doença, muito pouco se conhece sobre a saúde dos povos indígenas no Brasil, ainda mais se considerarmos a enorme diversidade sócio-cultural e de experiências históricas de interação com a sociedade nacional ${ }^{32}$.

Esse quadro de expansão demonstra uma tendência crescente e proeminente, dado que o período de 2010 a 2018 representa o maior quantitativo de publicações da revisão $(\mathrm{N}=211)$. Por meio desse mapeamento é possível notar a consolidação do campo de produção do conhecimento em saúde indígena ao longo dos últimos 20 anos (Gráfico 3).

No total da revisão, foram encontradas 35 temas (Gráfico 4) de acordo com as primeiras palavras-chave dos artigos: doenças infecto-parasitárias; nutrição; demografia; saúde bucal; saúde mental; medicinas tradicionais indígenas; genética de populações; doenças crônicas e não transmissíveis; atenção à saúde, política pública de saúde indígena e participação indígena. Com menos de três artigos aparecem temas diversos como saúde da mulher, sexualidade, câncer, oftalmologia, neurologia, cardiologia, farmacologia, entre outros.

As temáticas são diversas e correspondem a diferentes vertentes disciplinares, sendo que a maior quantidade de publicações sobre doenças infecciosas $(\mathrm{N}=142)$ advém das grandes áreas de medicina tropical e da epidemiologia; áreas que se entrecruzam na saúde coletiva, dado que a epidemiologia consiste em um dos seus pilares ${ }^{33}$. A nutrição, emerge como temática frequente e 


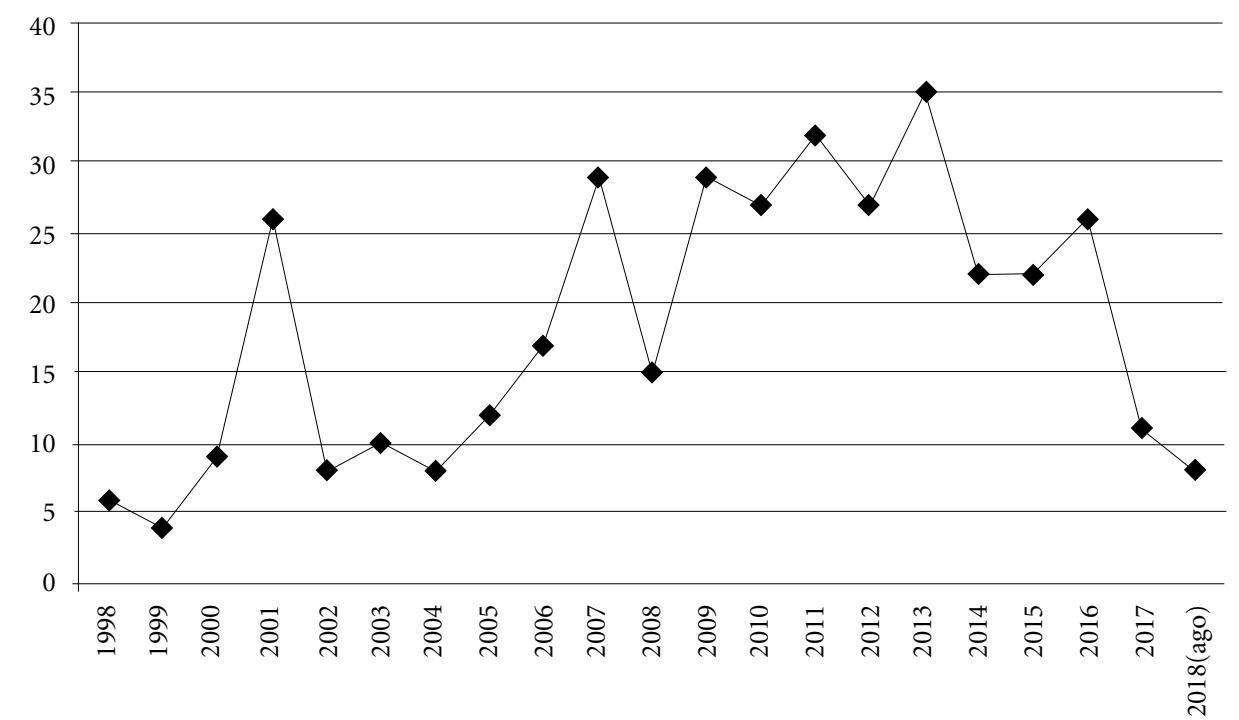

Gráfico 3. Distribuição (\%) da produção científica nos anos de 1998 a 2018 (N = 384).

Fonte: Elaboração própria.

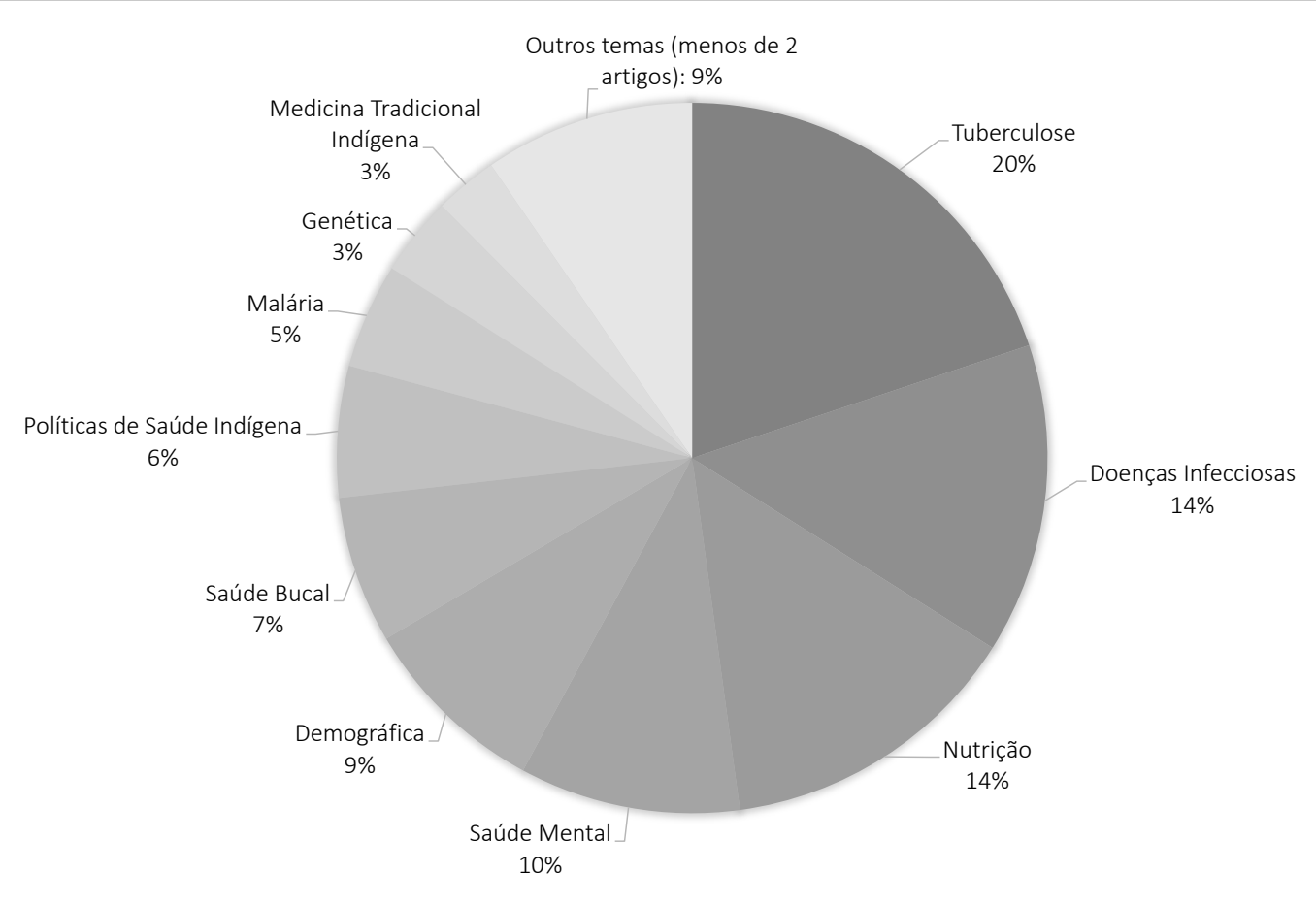

Gráfico 4. Distribuição dos artigos da área de saúde indígena $(\mathrm{N}=418)$ conforme temas por palavras-chave em todo o período analisado. 
relevante, em especial nos trabalhos acerca dos hábitos alimentares dos povos indígenas e avaliação das condições de crescimento e desenvolvimento da população ${ }^{34}$. Pesquisadores oriundos da Fiocruz e de universidades, principalmente de Mato Grosso do Sul e do Rio de Janeiro, realizaram os primeiros estudos sobre nutrição e povos indígenas. Ademais, foram responsáveis para a formação de quadro profissional na pós-graduação em saúde pública. Dos 58 artigos de nutrição, todos são de pesquisadores vinculados a grupos e linhas de pesquisa em saúde dos povos indígenas.

Os estudos sobre tuberculose foram separados do conjunto das doenças infecto-parasitárias, tendo em vista a emergência da questão, desde 1952, com as ações precursoras de Noel Nutels ${ }^{35}$. Dos 83 artigos localizados, 15 eram de periódicos da medicina tropical e os demais do campo da saúde coletiva, com destaque para um grupo da Fiocruz. Ressalta-se que atualmente há outros grupos de pesquisa que estudam especificamente a temática, devido a sua elevada prevalência nos povos indígenas.

Sobre demografia localizou-se 36 publicações, com maior concentração no ano de 2009 e na Revista Brasileira de Estudos Populacionais. Esse tema obtém destaque na produção da área após a implantação do Subsistema de Atenção à Saúde Indígena e o princípio de organização do sistema de informação com um módulo de demografia. Tal assunto ganhou impulso após 2010 quando o Censo do IBGE inseriu a identificação por raça/cor, mas ainda permanece um desafio o reflexo dessa coleta de informações nas publicações científicas.

Outras temáticas demonstram as importantes convergências da área de saúde indígena com a antropologia, como saúde mental e medicinas tradicionais indígenas, realizadas por antropólogos da saúde. Tais pesquisas se interessam tanto pelos hábitos considerados problemáticos do ponto de vista da saúde pública, como o consumo abusivo de álcool e drogas, quanto pelas percepções e práticas de autoatenção dos povos indígenas. Foram encontrados 27 artigos na área de antropologia e ciências sociais sobre temas diversos, desde avaliação das políticas de saúde, sistemas médicos tradicionais e participação indígena.

\section{Discussão}

Os povos indígenas do Brasil encontram-se em situação de grande vulnerabilidade socioeconô- mica e de saúde, que os colocam em desvantagem de oportunidades e de acesso a direitos em comparação aos demais cidadãos. Enquanto esta condição continua sendo uma temática de pesquisa, o protagonismo indígena na luta pelos seus direitos cresce nesse debate, inclusive, no meio acadêmico $^{36}$. A produção científica analisada demonstra que a academia colaborou ao dar visibilidade a essas desigualdades em saúde e às especificidades dessa população no acesso ao direito à saúde. Esses dados nos permitem iniciar um debate sobre o lugar da academia para a amplificação da agenda política da redemocratização do Brasil ${ }^{37}$.

Daniel Mundukuru, um dos maiores intelectuais indígenas da atualidade, destaca o protagonismo dos movimentos indígenas e contextualiza o apoio de entidades acadêmicas, pesquisadores e intelectuais no processo de transformação das legislações indígenas na década de 1970 e no desenvolvimento deste debate. $\mathrm{O}$ que resultou na Constituição Federal de 1988 e nos direitos atualmente consagrados ${ }^{38}$. A relação entre povos indígenas e pesquisadores é objeto de interesse de estudos que buscam descolonizar a ciência e a relação de poder estabelecida com grupos subalternos ${ }^{39}$.

Em convergência com outros estudos ${ }^{40,41}$, os achados indicam a considerável expansão da pesquisa em saúde dos povos indígenas no Brasil nas últimas décadas, seguindo o desenvolvimento do campo da saúde coletiva, ainda que com dinâmica própria. Tal crescimento é acompanhado da disseminação e distribuição em diversos campos disciplinares, dado que a saúde indígena envolve uma multiplicidade de domínios disciplinares que compõe seu modus operandi. Ou seja, se caracteriza tanto como um campo, quanto pela pulverização em diferentes campos. Ademais, abarca uma intensa sociodiversidade que se expressa nas especificidades socioculturais e linguísticas de cada população e nas diferenças no interior de cada grupo étnico.

O olhar sobre essa área do conhecimento na saúde coletiva revela diferentes campos disciplinares, que dialogam em torno da saúde dos povos indígenas. Se a lente fosse direcionada para cada campo - medicina, nutrição, medicina tropical, antropologia e políticas de saúde - veríamos que as pesquisas com povos indígenas aparecem como subtemas dos diferentes campos da saúde coletiva. Contudo, verifica-se que cerca de $40 \%$ das publicações são da área da Saúde Coletiva; ou seja, o montante da produção acadêmica sobre saúde indígena está inserida principalmente neste campo científico ${ }^{7,8,40}$. Tais dados revelam que 
a produção em saúde indígena reflete os debates próprios do campo da saúde coletiva no Brasil e das suas subáreas como a epidemiologia, as ciências sociais e humanas em saúde e a política e o planejamento em saúde.

Outro aspecto revelado pelo mapeamento sistemático diz respeito às transformações no desenvolvimento das temáticas e preocupações concernentes à saúde dos povos indígenas ao longo das décadas. Esses dados demonstram que a pauta de pesquisa em saúde indígena dialoga com as preocupações sociais, políticas e científicas de cada período. A linha do tempo na Figura 1 descreve essas mudanças.

Na linha do tempo nota-se a consolidação de temas e acumulação do conhecimento produzido, sendo que a última década concentra temas diretamente associados à implantação do Subsistema de Atenção à Saúde Indígena, tais como: "Atenção Diferenciada e Articulação de Sistemas Médicos"; "Agentes Indígenas de Saúde"; "Participação social"; "Transição epidemiológica e demográfica"; "Transição nutricional”; "Mortalidade infantil"; "doenças crônico-degenerativas"; "doenças infecto-parasitárias", entre outros. Essa evidência sugere que os pesquisadores e produtores de conhecimento na área são atores envolvidos, direta ou indiretamente, com as políticas públicas voltadas aos povos indígenas e às mudanças do perfil epidemiológico e nutricional dessa população.

Quanto às especificidades da produção de conhecimento na área da saúde indígena, a partir da década de 1990, nota-se que sua existência é mediada por relações entre saberes e práticas distintas em um mesmo campo de ação; seja do ponto de vista das políticas públicas, seja da perspectiva epistemológica. Tal característica foi denominada por Foller de zona de contato ${ }^{42}$. Isso significa dizer que nesta zona existe uma assimetria que envolve intensos processos de negociação e relações de poder entre a biomedicina - representada pela normatividade das políticas de saúde e a presença de profissionais de saúde nos territórios indígenas - e as concepções cosmológicas e relações socioculturais e políticas no interior da vida comunitária dos povos indígenas e da sociedade envolvente ${ }^{43}$.

A biomedicina é compreendida como um recurso de saber-poder colonizador e, trazida para este contexto, se revela na relação entre Estado, ciência e povos indígenas. Quer dizer, mesmo com a mudança da perspectiva constitucional a respeito dos povos indígenas - ao transformar a concepção tutelar pelo reconhecimento e auto- nomia da diversidade étnica, com a inclusão da diretriz da atenção diferenciada no subsistema -, o saber colonial pode se fazer presente pelo formato do cuidado e da atenção à saúde, onde verificamos o pleno exercício do biopoder ${ }^{44}$. Souza-Lima ${ }^{10}$ identifica, na gestão colonial das desigualdades, o movimento de perpetuação e modernização da lógica tutelar por meio de organismos e práticas que circunscrevem e subjugam populações; ainda que sob o ideário salvacionista e de valor à vida própria do discurso biomédico. Para o autor, o cerne dos poderes e saberes de ordem colonial que se condensaram em torno da prática indigenista tem origens históricas distintas. Mas, se concentram no propósito da conquista de espaços territoriais como ideológicos, socioculturais e simbólicos.

Garnelo $^{18}$ situa que a lógica colonial e integracionista, que estigmatiza a população como forma de manutenção da proteção estatal e do poder tutelar, ainda subjaz na promoção de direitos de cidadania. Nas suas palavras:

No caso das minorias étnicas, embora a cidadania represente um direito e um tipo de proteção social, ela também pode significar uma forma de homogeneizar o mundo indígena aos modos de vida da sociedade nacional, podendo ainda induzir à adoção de valores e comportamentos do grupo social hegemônico em detrimento da diferenciação étnica ${ }^{18}$

Ao compreender a área de estudos em saúde dos povos indígenas como uma produção numa "zona de contato", reconhecemos sua capacidade de ultrapassar fronteiras e dialogar com diversas perspectivas disciplinares. O que designa sua manutenção e crescimento no interior do campo da Saúde Coletiva e em outros campos do conhecimento, como a Antropologia. Como revela a análise de Teixeira e Silva acerca das teses e dissertações de antropologia sobre saúde indígena, esses estudos se localizam nas fronteiras entre campos disciplinares bem constituídos ${ }^{31}$. A área de antropologia médica e da saúde possui um papel fundamental, por buscar compreender e explicar as relações interétnicas que permeiam o contexto da saúde, assim como a construção, interpretação e intervenção nos corpos e no processo de saúde e doença ${ }^{11}$.

Langdon e Follér ${ }^{45}$ descrevem as preocupações em torno das condições de saúde e adoecimento das populações através de abordagem estritamente antropológica, a partir da criação de disciplinas em cursos de pós-graduação, reuniões científicas e grupos de estudo sobre o tema. Follér ${ }^{42}$ assinala ainda a importância da atuação 
- Interesse voltado para as grandes epidemias que assolavam os povos indígenas e em verificar o grau de especificidade ou generalidade dos povos indígenas em relação a outras populações;

- Emergência da saúde coletiva e discussões sobre políticas públicas específicas para esses povos;

- Período da implantação do Subsistema de Saúde Indígena, com predominância de conteúdos sobre os desafios da implementação dos serviços de saúde

- Consolidação da saúde indígena como uma área específica do conhecimento e com acúmulo de produção científica

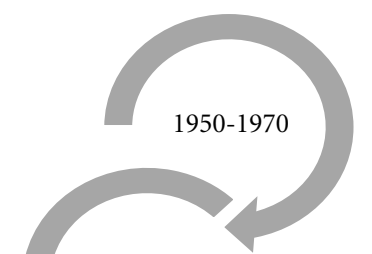

1970-1980

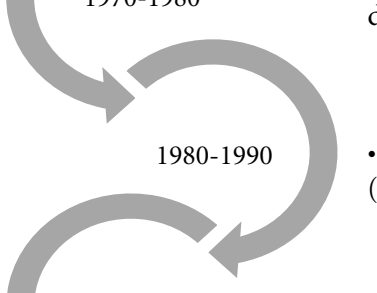

1990-2000

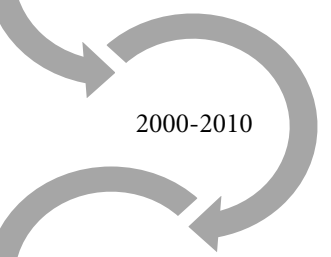

2010- Atual
- Preocupações em torno da área de medicina tropical com o desenvolvimento dos estudos sobre agentes etiológicos das doenças

- Criação do Sistema Único de Saúde (SUS)

- Destaque das temáticas específicas e de maior diálogo entre áreas de conhecimento e com as realidades dos serviços de saúde, tais como, nutrição, mortalidade Infantil, tuberculose, política nacional de saúde, sistemas médicos tradicionais;

Figura 1. Linha do tempo sobre as temáticas predominantes por década sobre saúde dos povos indígenas, de 1950 a 2018.

Fonte: Elaboração própria.

de cientistas sociais e antropólogos nas ações e serviços de saúde como modo de flexibilizar fronteiras epistemológicas e de práticas médicas em um contexto intercultural, em que se relacionam a biomedicina e os saberes e práticas tradicionais em saúde dos povos indígenas.

Outra vertente do campo da Saúde Coletiva, que aparece como potencializador da área de saúde indígena, é a formulação e implementação das políticas públicas em saúde direcionadas à proteção de grupos específicos. Segundo Garne$10^{19}$, a focalização é construída com o objetivo de atingir uma população em específico e a universalização prevê o conjunto da população nacional de forma indiscriminada. Ambas as abordagens não são necessariamente excludentes e convivem em disputas e tensionamentos, podendo ser complementares, como no caso das políticas de saúde indígena. A área de saúde indígena contribuiu assim para os debates sobre focalização versus universalização das políticas sociais, conforme o referencial conceitual da equidade e um dos pilares do SUS.

$O$ conceito de equidade à luz do Estado Brasileiro significa tratar o diferente de modo diverso em busca da igualdade de direitos na perspectiva da justiça social ${ }^{46}$. Vieira-da-Silva e Almeida Fi1 ho ${ }^{47}$ discutem os diferentes conceitos de equidade e terminologias utilizadas como sinônimos, tais como iniquidade, desigualdade, entre outras, ao longo da história e de sua introdução no campo da saúde.

Desse modo, a equidade torna-se um conceito caro às políticas públicas diferenciadas, ao trazer para o campo da saúde pública a necessidade de uma atenção específica às populações diversas, em respeito às diversidades étnicas, socioculturais e regionais. Por se tratar de uma prerrogativa legal e moral, é um conceito que traz impacto importante para a dimensão científica, pois dire- 
ciona um tratamento qualificado frente às diversidades étnicas no universo das ciências da saúde e das análises a respeito do SUS ${ }^{47}$.

Os resultados deste mapeamento revelam que a implementação de políticas e serviços públicos de saúde indígena contribuíram para a emergência das temáticas estudadas, demonstrando como o campo científico está em constante interface com o político. Ao mesmo tempo, é afetado pelas relações mais amplas, sendo necessário apontar as desigualdades existentes dentro do próprio campo científico no quesito priorização para políticas de fomento.

No caminho indicado por Bourdieu, cabe salientar o poder de influência que as evidências científicas podem implicar nas decisões políticas e políticas de Estado ${ }^{21}$. Ou mesmo, avaliar se a condução das políticas é ou não capaz de dialogar com a produção do conhecimento em saúde dos povos indígenas. Ainda que este mapeamento não tenha a pretensão de resolver esse problema, do ponto de vista investigativo, essa associação surge como lacuna a ser desenvolvida por estudos futuros.

\section{Considerações finais}

Verificamos que nas últimas décadas a tema da saúde indígena se consolida nos meios acadêmicos e nas políticas públicas, refletindo a nova conjuntura das relações entre Estado brasileiro e povos indígenas. Parte relevante dessa produção se localiza no campo da Saúde Coletiva, implicado com o projeto político da Reforma Sanitária e a criação do SUS. A diversidade das temáticas abordadas nos artigos e a sua presença em vários periódicos, revelam o quanto essa área do conhecimento consolida-se na Saúde Coletiva. Todavia, também se dissemina por diferentes campos disciplinares, sendo extremamente potente para abordagens complexas e interdisciplinares.

Por meio da descrição dos principais temas e enfoques da produção científica sobre saúde indígena, constatamos que o crescimento dessa produção está diretamente relacionado à sua inserção no cenário sociocultural, político e operacional das políticas públicas em saúde no Brasil. $\mathrm{Tal}$ inserção reflete os diálogos dessa produção com o cenário de inúmeros desafios vivenciados pela implementação do subsistema de saúde indígena, pelas desigualdades em saúde que afetam os povos indígenas e pelas importantes transformações dessa população em termos demográficos, epidemiológicos e nutricionais. A despeito das especificidades dessas populações, a produção de conhecimento dessa área pode contribuir para o enfrentamento das iniquidades que incidem nas condições de saúde e de vida da população brasileira.

Compreendemos que o conhecimento que baliza a produção científica sobre saúde indígena é intrinsecamente comprometido com horizontes politicamente implicados nas realidades estudadas e com a visibilidade de populações marginalizadas. O que geralmente pode ocorrer em tensões com as agendas governamentais. É possível afirmar, portanto, que a emergência e a constituição da área de estudos de saúde dos povos indígenas permite a coexistência no ambiente científico tanto de um modelo hegemônico de construção do saber sobre saúde e doença, quanto de uma arena que permite o contra fluxo e a crítica a essa hegemonia.

Observamos que as preocupações levantadas pelas publicações se aproximam das demandas da sociedade civil organizada, do movimento indígena ao movimento sanitarista e, consequentemente, se alinha ao caminho da democratização de direitos de populações desfavorecidas pelo Estado. O que favorece a própria apropriação do conhecimento científico pela sociedade civil ao torná-la não só pública, quanto passível de ser aplicada a processos de melhoria das condições de vida das populações indígenas. Em uma perspectiva histórica, assim como o movimento de reforma sanitária trouxe essa marca para o SUS, que se concretizou em um sistema de saúde fruto da sociedade civil organizada, o movimento indigenista em articulação com diferentes sujeitos, incluindo pesquisadores, forjaram a criação de um subsistema de atenção à saúde indígena. Sabemos, contudo, que publicizar o conhecimento científico não é suficiente para a sua devida transposição além dos muros da academia.

O processo histórico de extermínio, violências e resistência dos povos indígenas no Brasil e as consequentes epidemias, mortes, invasões territoriais e transformações nos seus modos de vida nos levam a apontar a relevância das discussões sobre o papel do Estado e da produção do conhecimento na construção de políticas públicas nas mais diferentes áreas direcionadas a esses povos. Papel que é ao mesmo tempo necessário e problemático, sobretudo, quando pensados e executados de uma maneira pouco compartilhada com as populações a que assistem. Vários aspectos inserem-se nesta questão, sendo extensa a discussão a respeito das políticas e dos demais projetos intervencionistas nas terras indígenas 
e que, muitas vezes, não correspondem às reais necessidades dos povos indígenas. Inúmeros estudos atestam, inclusive, os desafios em torno da dificuldade de diálogo entre pesquisadores, gestores, profissionais de saúde e povos indígenas.

Existem constantes críticas dos povos indígenas quanto às práticas de pesquisadores que estudam em suas áreas e que não retornam às populações os conhecimentos produzidos. Todavia, vemos na saúde indígena esforços para a criação de vínculos próximos entre pesquisadores e os povos, seja com a contrapartida política na oferta de ações de saúde ou em atividades de pesquisa-extensão em áreas indígenas. E, no momento atual, com o desenvolvimento das políticas de ação afirmativa, a implementação de processos seletivos específicos e cotas para indígenas nos cursos de nível superior e na pós-graduação, inicia-se a formação de pesquisadores indígenas na área da saúde e afins. Como perspectiva do futuro da área da saúde indígena, imaginamos que esses pesquisadores indígenas irão provocar novas transformações e mudanças no campo.

Mesmo sendo um contingente populacional pequeno, se comparado à população nacional como um todo, a diversidade étnica dos povos indígenas nos revela os desafios no âmbito da equidade, justiça social e direitos humanos. Inúmeros são as problemáticas em torno das condições de vida e sobrevivência dos povos indígenas, especialmente no campo da saúde. Nesse sentido, a diversidade pode ser vista como uma oportunidade de abranger horizontes teóricos e metodológicos.
Concordando com a afirmação de Boaventura de Souza Santos ${ }^{48}$, Todo conhecimento científico visa constituir-se em senso comum, verificamos que na saúde indígena, ao mesmo tempo em que o senso comum dominante da sociedade nacional é o preconceito e a discriminação perante os povos indígenas, o conhecimento científico que emerge na produção acadêmica da saúde indígena busca demonstrar um outro ponto de vista válido, de modo a se diferenciar desse corpo social.

Nesse sentido, o presente artigo objetivou compreender o panorama sobre a produção do conhecimento científico sobre saúde dos povos indígenas e sua interface com a garantia de direitos e diminuição das iniquidades em saúde. Como limitação, entendemos que uma análise mais aprofundada sobre os conteúdos dos artigos e suas perspectivas epistemológicas, teóricas e metodológicas, pode avançar no debate; bem como a consideração de outras publicações como livros, artigos de livros, teses, dissertações e trabalhos completos de eventos científicos. Ainda que existam análises e levantamentos anteriores, que visavam dar conta desta lacuna, a principal contribuição deste artigo consiste em trazer evidências sobre a inserção da área de estudos de saúde indígena no campo da Saúde Coletiva no Brasil, conforme sinalizado pelos próprios pesquisadores do campo. E, sendo parte, nos interessa avançar na compreensão do quanto a saúde indígena reflete mudanças do campo maior e incide sobre ele em constante movimento e inovações científicas e políticas.

\section{Colaboradores}

JF Kabad: Responsável pela pesquisa, concepção e desenho do artigo, redação e revisão do texto. ALM Pontes: Co-orientadora da pesquisa, concepção e desenho do artigo, redação e revisão do texto. S Monteiro: Orientadora da pesquisa, adequação do desenho do estudo, redação e revisão do texto. 


\section{Referências}

1. Instituto Brasileiro de Geografia e Estatística (IBGE). Os indígenas no Censo Demográfico 2010 - primeiras considerações com base no quesito cor ou raça. Rio de Janeiro: IBGE; 2012.

2. Marinho GL, Santos RV, Pereira NOM. Classificação dos domicílios "indígenas" no Censo Demográfico 2000: subsídios para a análise de condições de saúde. R. bras. Est. Pop. 2011; 28(2):449-466.

3. Pereira NOM. Importância dos censos nacionais no conhecimento da demografia e da saúde dos indígenas do Brasil. Rio de Janeiro: Instituto Brasileiro de Geografia e Estatística (IBGE); 2009.

4. Guimarães MCS, Novaes SC. Autonomia Reduzida e Vulnerabilidade: Liberdade de Decisão, Diferença e Desigualdade. Revista Bioética 2009; 7(1):1-3.

5. Pagliaro H, Azevedo MM, Santos RV, organizadores. Demografia dos povos indígenas no Brasil. Rio de Janeiro: Editora Fiocruz, Associação Brasileira de Estudos Populacionais; 2005.

6. Coimbra Júnior C, Santos R. Saúde, minorias e desigualdade: algumas teias de inter-relações com ênfase nos povos indígenas no Brasil. Cien Saude Colet 2000; 5(1):125-132.

7. Garnelo LM, Macedo G, Brandão LC. Os Povos Indígenas e a Construção das Políticas de Saúde no Brasil. Brasília: Organização Pan-Americana de Saúde (OPAS); 2003.

8. Paim JS, Almeida Filho N. Saúde coletiva: uma "nova saúde pública" ou campo aberto a novos paradigmas? Rev Saude Publica 1998; 32(4):299-316.

9. Giovanella LM, Mendonça MHM. Atenção Primária à Saúde: seletiva ou coordenadora dos cuidados? Rio de Janeiro: CEBES; 2012

10. Souza-Lima AC. Revisitando a Tutela: questões para se pensar as políticas públicas para povos indígenas. In: Souza-Lima AC, organizador. Saúde Indígena em Perspectiva: explorando suas matrizes históricas e ideológicas. Rio de Janeiro: Fiocruz; 2014. p. 27-58.

11. Langdon EJ. Uma avaliação crítica da atenção diferenciada e a colaboração entre antropologia e profissionais de saúde. In: Langdon EJ, Garnelo L, organizadoras. Saúde dos Povos Indígenas: reflexões sobre antropologia participativa. Rio de janeiro: Contra Capa, Associação Brasileira de Antropologia; 2004. p. 33-52.

12. Cardoso MD. Políticas de saúde indígena no Brasil: do modelo assistencial à representação política. In: Langdon EJM, Cardoso MD, organizadoras. Saúde indígena: políticas comparadas na América Latina. Florianópolis: Editora da UFSC; 2015. p. 83-106,

13. Garnelo L, Maquiné A. Financiamento e gestão do subsistema de saúde indígena: considerações à luz dos marcos normativos da administração pública no Brasil. In: Langdon EJ, Cardoso MD, organizadoras. Saúde Indígena: políticas comparadas na América Latina. Florianópolis: Editora da UFSC. 2015; p. 107-144

14. Teixeira CC, Garnelo L. Saúde Indígena em Perspectiva: explorando suas matrizes históricas e ideológicas. Rio de Janeiro: Fiocruz; 2014.
15. Luz Madel T. Complexidade do campo da Saúde Coletiva: multidisciplinaridade, interdisciplinaridade, e transdisciplinaridade de saberes e práticas - análise sócio-histórica de uma trajetória paradigmática. Saude Soc 2009; 18(2):304-311.

16. Kabad JF, Bastos JL, Santos RV. Raça, cor e etnia em estudos epidemiológicos sobre populações brasileiras: revisão sistemática na base PubMed. Physis 2012; 22(3):895-918.

17. Monteiro S, Sansone L. Etnicidade na América Latina: um debate sobre raça, saúde e direitos reprodutivos. Rio de Janeiro: Editora Fiocruz; 2004.

18. Garnelo LM. Política de Saúde dos Povos Indígenas no Brasil: Análise Situacional do Período de 1990 a 2004. Universidade Federal de Rondônia, 2004. [acessado 2019 Maio 20]. Disponível em: http://www.cesir.unir. br/pdfs/doc9.pdf

19. Garnelo L. O SUS e a Saúde Indígena: matrizes políticas e institucionais do Subsistema de Saúde Indígena. In: Teixeira CC, Garnelo L, organizadoras. Saúde Indígena em Perspectiva: explorando suas matrizes históricas e ideológicas. Rio de Janeiro: Editora Fiocruz; 2014. p. 107-142.

20. Paim JS. Desafios para a saúde coletiva no século XXI. Salvador: EDUFBA; 2006.

21. Bourdieu P. O campo científico.In: Ortiz R, organizador. Pierre Bourdieu: Sociologia. São Paulo: Ática; 1983. p. 122-125.

22. Bourdieu P. O Poder Simbólico. Rio de Janeiro: Editora Bertrand Brasil; 2010.

23. Pereira GRM, Catani AM. Espaço social e espaço simbólico: introdução a uma topologia social. Perspectiva 2002; 20(n. esp.):107-120.

24. Latour B. Ciência em ação: como seguir cientistas e engenheiros sociedade afora São Paulo. São Paulo: UNESP; 2000.

25. Instituto de Estudos Socioeconômicos (INESC). Movimento indígena impede retrocessos no atendimento à saúde. [acessado 2019 Maio 20]. Disponível em: https://www.inesc.org.br/movimento-indigena-impede -retrocessos-no-atendimento-a-saude/

26. Silva EL, Pinheiro LV. A produção do conhecimento em ciência da informação no brasil: uma análise a partir dos artigos científicos publicados na área. Intexto 2008; 2(19):1-24.

27. Universidade Estadual Paulista (UNESP). Tipos de revisão de literatura. Botucatu: UNESP; 2015.

28. Buchillet D. Bibliografia crítica da saúde indígena no Brasil. Quito: Abya-Yala; 2007.

29. Ribeiro D. Atlas Histórico do Brasil. CPDOC/FGV. [acessado 2019 Maio 20]. Disponível em: https://atlas. fgv.br/verbete/ 4555

30. Souza R, Cardoso AM, Garnelo L, Coimbra Júnior CEA, Chaves MBG. Saúde dos Povos Indígenas e Políticas Públicas no Brasil. In: Giovanella L, Escorel S, Lobato, LVC, Noronha JC, Carvalho AI, organizadores. Políticas e Sistemas de Saúde no Brasil. Rio de Janeiro: Editora Fiocruz; 2013. p. 911-932.

31. Teixeira CC, Silva CD. Antropologia e saúde indígena: mapeando marcos de reflexão e interfaces de ação. Anuário Antropológico 2013; 38(1):35-37. 
32. Santos RV, Escobar AL. Editorial - Saúde dos povos indígenas no Brasil: perspectivas atuais. Cad Saude Publica 2001; 17(2):258-259.

33. Almeida Filho N. Bases históricas da Epidemiologia. Cad Saude Publica 1986; 2(3):304-311.

34. Lício JSA. Estado Nutricional de Crianças Indígenas no Brasil: Uma Revisão Sistemática da Literatura Científca [dissertação]. Rio de Janeiro: Fiocruz; 2009.

35. Costa DC. Política indigenista e assitência à saúde Noel Nutels e o serviço de unidades sanitárias aéreas. Cad Saude Publica 1987; 3(4):388-401.

36. Luciano GJS, Oliveira JC, Hoffman MB. Olhares indígenas contemporâneos. Brasília: Centro Indígena de Estudos e Pesquisas; 2010.

37. Souza Lima AC. O exercício da tutela sobre os povos indígenas: considerações para o entendimento das políticas indigenistas no Brasil contemporâneo. Revista de Antropologia 2012; 55:781.

38. Danner F, Dorrico J, Danner F. Indígenas em movimento. Literatura como ativismo. Remate de Males 2018; 38(2):919-959.

39. Vargas VLF, Castro I. Os pesquisadores de índios e os índios pesquisadores. Revista Virtual Outros Tempos 2013; 10:16.

40. Mendes AM, Leite MS, Langdon EJ, Grisotti M. O desafio da atenção primária na saúde indígena no Brasil. Rev Panam Salud Publica 2018; 42:e184.

41. Nunes ED. Pós-graduação em Saúde Coletiva no Brasil: Histórico e Perpectivas. Physis 2005; 15(1):13-38.

42. Foller ML. Intermedicalidade: a zona de contato criada por povos indígenas e profissionais de saúde. In: Langdon EJ, Garnelo L, organizadoras. Saúde dos Povos Indígenas: reflexões sobre antropologia participativa. Rio de Janeiro: Contra Capa, Associação Brasileira de Antropologia; 2004. p. 129-147.

43. Langdon EJ, Wilk FB. Antropologia, saúde e doença: uma introdução ao conceito de cultura aplicado às ciências da saúde. Rev. Latino-Am. Enfermagem [Internet]. maio-jun 2010.[acessado 2020 Mar 22]; 18(3): [9 Telas].Disponível em: http://www.scielo.br/pdf/rlae/ v18n3/pt_23

44. Silva DR. Biopoder na concepção de Michel Foucault: o poder do Estado no controle da sociedade. Periagoge 2018; 1(1):1-13.

45. Langdon EJ, Foller ML. Antropology of Health in Brazil: a border discourse. Med Antropol 2012; 31(1):4-28.

46. Whitehead $\mathrm{M}$. The concepts and principles of equity in health. Int J Health Serv 1992; 22(3):429-445.

47. Vieira-da-Silva LM, Almeida Filho N. Equidade em saúde: uma análise crítica de conceitos. Cad Saude Publica 2009; 25(Supl. 2):S217-S226.

48. Santos BS. Um Discurso Sobre as Ciências. 16a ed. Porto: B. Sousa Santos, Edições Afrontamento; 2010. 
